\title{
SOBRE EL OBJETO Y LA VIABILIDAD DE LA RESTAURACIÓN AMBIENTAL
}

\author{
Alfredo Ollero Ojeda \\ Dpto. de Geografía y Ordenación del Territorio, Universidad de Zaragoza \\ aollero@unizar.es
}

Resumen: Frente al concepto más empleado de restauración ecológica, se propone el de restauración ambiental como el proceso de auto-recuperación de un sistema natural (de sus procesos, estructura, funciones, territorio, dinámica y resiliencia) a partir de la eliminación de los impactos que lo degradaban hasta alcanzar un funcionamiento natural y autosostenible. El paradigma de restauración debe mantenerse en ciencia y educación, como marco teórico, pero para recuperar sistemas naturales complejos y fuertemente degradados es poco viable. Es más práctico optar por medidas de rehabilitación basadas en objetivos alcanzables y con seguimiento científico. Debe evitarse el empleo del término restauración como marketing y las acciones de restauración y rehabilitación como negocio o estrategia de maquillaje.

Palabras clave: restauración ambiental, rehabilitación, presión humana, situación de referencia, trayectoria, naturalidad.

\begin{abstract}
An approach to the target and feasibility of environmental restoration. Environmental restoration is proposed instead of "ecological restoration". This concept is understood as the process of self-recovery of a natural system (its processes, structure, functions, territory, dynamics and resilience) since the removal of the impacts to a natural and sustainable functioning. The paradigm of restoration should remain as a theoretical framework in science and education; however, is not feasible to restore complex and heavily deteriorated natural systems. It is more sensible to use rehabilitation measures based on achievable goals and scientific monitoring. Finally, it should be avoided the use of the term "restoration" as marketing, and the restoration and rehabilitation actions as business or cosmetic strategy.
\end{abstract}

Recibido: 30-9-10. Aceptado: 3-11-11. 
Keywords: environmental restoration, rehabilitation, human pressure, reference state, trajectory, naturalness.

\section{Introducción}

La restauración ambiental es el más avanzado de los procesos de gestión de un sistema natural. Es un proceso fundamentalmente recuperador, pero también corrector y preventivo. Es el último paso, el más avanzado, pero sólo es factible en situaciones de gestión ambiental sostenible, resultando difícil o imposible en sociedades incapaces de renunciar a su imparable consumo de recursos. En estos casos la restauración es muy urgente y necesaria, pero resulta inaplicable por la imposibilidad de eliminar, o siquiera reducir, las presiones. En estos modelos económicamente "avanzados" y ambientalmente insostenibles puede aspirarse a lo sumo a la rehabilitación, al logro de pequeñas mejoras, o a la protección de pequeños enclaves inconexos y relictos. Así, en líneas generales, se han producido algunos éxitos puntuales de restauración en sistemas naturales pequeños y relativamente cerrados (humedales endorreicos, por ejemplo), pero no se han podido llevar a cabo procesos de auténtica restauración en sistemas más extensos, abiertos y complejos.

Es precisamente en estas sociedades de consumismo y grave deterioro ambiental donde han surgido preocupaciones científicas y sociales de restauración, en muchas ocasiones obligadas a quedarse en la teoría o las buenas intenciones. Desde el ecologismo y las ciencias ambientales se ha desarrollado el sentimiento de la belleza y el valor de lo natural y la responsabilidad de su conservación (González del Tánago y García de Jalón, 2007). Es un deber conservar y mejorar nuestro entorno para traspasarlo a las generaciones futuras en buen estado. Tenemos incluso la obligación moral de renaturalizar muchos ambientes dañados por una larga historia de insensibles obras y actuaciones sobre el medio (Soar y Thorne, 2001). Incluso muy recientemente se ha comenzado a apreciar los sistemas naturales activos, dinámicos, heterogéneos, complejos, inestables, temporales, fluctuantes, irregulares, imprevistos, en continuo cambio, la estética de lo cambiante y salvaje, rechazando la domesticación y simplificación propugnadas por la sociedad del consumo y el bienestar.

Así, la restauración ambiental se ha convertido en un gran reto de nuestro tiempo, pero se está quedando en quimera irrealizable, sería necesario un profundo cambio de mentalidades en lo territorial y ambiental hacia la reducción del consumo y hacia la sostenibilidad. Quizás la sociedad no esté preparada para ese cambio, pero es interesante constatar que en estas sociedades "avanzadas" haya surgido un importante yacimiento de empleo y nicho de mercado en torno a la "restauración", así como un 
motivo de prestigio y factor de localización para las "áreas restauradas" (Gómez Orea, Dir., 2004). No suele tratarse de auténtica restauración, sino de prácticas de maquillaje que forman parte de procesos urbanísticos y especulativos. Es el marketing de la palabra "restauración", utilizada en demasía, con absoluta falta de propiedad y con objetivos no ambientales (Elliot, 1982; Katz, 2003).

Ante el reto o la utopía de la auténtica restauración, han ido apareciendo en los últimos años, principalmente desde el ámbito de la restauración fluvial (Dufour y Piégay, 2009), nuevas tendencias posibilistas que renuncian al imposible retorno a estados naturales y abogan por una restauración basada en objetivos concretos y factibles, incompleta pero que responda a criterios de naturalidad y aporte beneficios a la sociedad. Es un planteamiento interesante que, aunque implica renunciar a ideales, abre muchas puertas de actuación, si bien requiere diseñar con mucho cuidado los programas de mejora, por el riesgo de caer en el puro marketing perdiendo los objetivos ambientales.

\section{Qué es restauración ambiental}

Restaurar es restablecer o recuperar un sistema natural a partir de la eliminación de los impactos que lo degradaban y a lo largo de un proceso prolongado en el tiempo, hasta alcanzar un funcionamiento natural y autosostenible. Un sistema natural restaurado habrá recuperado:

- sus procesos naturales y todas las interacciones entre sus elementos y con otros sistemas,

- su estructura, es decir, todos sus componentes y flujos en toda su complejidad y diversidad,

- sus funciones dentro del sistema Tierra (por ejemplo: transporte, almacén, regulación, hábitat...),

- su territorio, es decir, el espacio propio y continuo que debe ocupar para desarrollar todos sus procesos y funciones,

- su dinámica natural a lo largo del tiempo,

- su resiliencia o fortaleza frente a futuros impactos, su capacidad de auto-regulación y auto-recuperación

- y, por tanto, todos los bienes y servicios que aporta a la sociedad.

En suma, el proceso de restauración debe lograr naturalidad, funcionalidad, dinamismo, complejidad, diversidad y resistencia para el sistema natural. La auténtica restauración es, por tanto, auto-restauración. El sistema debe ser capaz de recuperarse 
solo progresivamente, a lo largo de un proceso, desde el momento en que se eliminen los impactos.

Es muy importante señalar que un sistema natural nunca puede volver a su estado original previo a las alteraciones que ha sufrido, ya que ha pasado un tiempo y el sistema ha estado integrado en una trayectoria (Turner et al., 1993), en una dinámica temporal integrada con la de otros sistemas. Restaurándolo se le puede llevar a un estado natural equivalente o parecido al original previo, pero nunca idéntico, porque muchos factores, elementos y procesos habrán cambiado durante esa trayectoria, incluso sin intervención humana (figura 1).

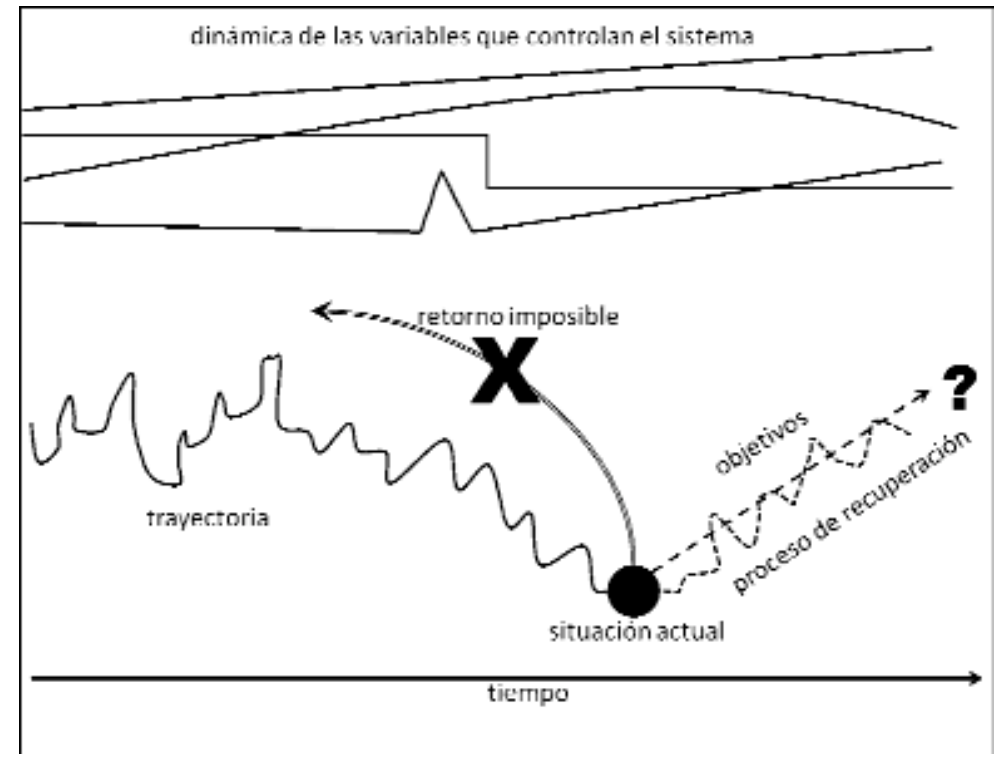

Figura 1. Trayectoria, retorno imposible y proceso de recuperación (adaptado de Dufour y Piégay, 2009).

\section{Discusión}

\section{1. ¿Restauración ambiental o restauración ecológica?}

Restauración ambiental y restauración ecológica pueden considerarse sinónimos, empleándose según la formación o profesión del que los utiliza. En la actualidad está más extendido el término "restauración ecológica", fomentado desde la Ecología y la 
Biología. Por ejemplo, realizada una búsqueda en Google Scholar (16-08-2010), se han encontrado 176 trabajos académicos en español, 56 en francés y 2.850 en inglés en cuyo título aparece la expresión "restauración ecológica", mientras los títulos con "restauración ambiental" son 93 en español, 1.970 en inglés y sólo 5 en francés.

Sin embargo, en este artículo se defiende el concepto de "restauración ambiental" por ser más abierto y generalista, entendiendo que la "restauración ecológica" tiene un sesgo hacia los objetivos de biodiversidad y en ocasiones adolece de déficits en ciertas perspectivas como la geomorfológica. Además, en muchos casos no es la biodiversidad la propiedad más valiosa de un sistema natural. Existe aquí un conflicto interprofesional que se manifiesta en todo el ámbito del conservacionismo y de las ciencias ambientales. Es también por ello que en este artículo se prefiere hablar de "sistemas naturales" frente a "ecosistemas".

En las definiciones de restauración ecológica es evidente la importancia de lo biológico. Por ejemplo, Sánchez (2005) la define como la práctica de acciones orientadas a propiciar una trayectoria de restablecimiento de un ecosistema previamente alterado, en compatibilidad con las condiciones actuales y con la historia biológica del entorno, de manera que consiga una recuperación significativa de sus atributos, rasgos estructurales, procesos y funciones, para que sea autosostenible.

\section{2. ¿Restauración utópica o rehabilitación realista?}

Los objetivos de la restauración no deben ser belleza ni recreo ni estabilidad, como es frecuente, ni simplemente cumplir con la legalidad. Restaurar no es estabilizar, ni revegetar, ni ajardinar, ni urbanizar, ni maquillar, ni camuflar. Por ejemplo, la restauración paisajística no es auténtica restauración, ya que se centra en el aspecto del sistema natural o de alguno de sus elementos, y no en la funcionalidad o dinámica del mismo, no persigue un objetivo natural. Las técnicas de bioingeniería o ingeniería natural, cada vez más extendidas, tampoco deben asociarse con la auténtica restauración, ya que generalmente se emplean para objetivos estéticos o de estabilización.

Por tanto, hay una restauración auténtica y muchas posibles mejoras parciales, a las que conviene no llamar restauración, sino rehabilitación, restablecimiento, remediación, mitigación, naturalización, creación, acondicionamiento, adecuación, etc., según sus caracteres. Parece que frente a la auténtica pero muchas veces utópica restauración se va consolidando el concepto de rehabilitación, como el conjunto de medidas factibles para la mejora, sin poder llegarse a la recuperación total.

Mientras la restauración es un paradigma teórico, puro, que responde exclusivamente a objetivos ambientales, la rehabilitación es una práctica que responde tam- 
bién a esos objetivos pero con las limitaciones que imponen las presiones humanas y en consenso con la sociedad, siendo definida por los gestores. La rehabilitación es, por tanto, una estrategia de gestión frente a la restauración, que es exclusivamente y estrictamente conservacionista. Ambas cuentan con una base científica, pero la rehabilitación es fundamentalmente técnica. La restauración es pasiva, ya que el trabajo lo realiza el propio sistema natural, una vez eliminados los impactos, pero la rehabilitación en muchos casos se considera activa, ya que emplea técnicas y estructuras concretas para acelerar los procesos.

En definitiva, la auténtica restauración es extremadamente difícil en la práctica por dos razones fundamentales:

- Porque muchas actividades humanas son incompatibles con los sistemas naturales y deberían ser modificadas o trasladadas, lo cual es social y económicamente muy complejo. La auténtica restauración constituiría toda una "revolución". Porque hay dos requisitos básicos para restaurar y la sociedad debería estar dispuesta a asumirlos: eliminar o reducir al mínimo las causas del deterioro y reconsiderar las actividades humanas que generaron esas causas. Sin embargo, los intensos procesos de urbanización y especulación siguen provocando un incremento continuo en el deterioro ambiental. Mientras se estén restaurando algunos espacios, muchos más estarán siendo alterados por acciones humanas. La correcta restauración está muy alejada de las actuales demandas sociales de domesticación del medio, urbanización y desnaturalización. Probablemente mientras la sociedad no cambie sus hábitos de consumo la restauración será una labor inútil.

- Porque en la mayoría de los casos y ámbitos de restauración no pueden encontrarse estados naturales de referencia en el pasado. La intervención humana ha sido extensa, compleja y duradera, y ha ido perturbando a los sistemas naturales directamente, pero también de muchas formas indirectas muy difíciles de identificar y cuantificar. No es suficiente con volver, por ejemplo, a la situación preindustrial. Es imposible saber en qué momento del pasado un sistema se encontró en su mejor estado. También hay determinados ecosistemas que justo en los periodos de mayor intervención humana es cuando han contado con mayor biodiversidad y complejidad. A esto hay que unir la realidad de que es imposible reproducir una situación pasada porque todo ha seguido una trayectoria (figura 1). La naturaleza es producto en cada momento de condiciones que no se repetirán. Y en el proceso de recuperación nunca se repetirá exactamente igual la trayectoria de la sucesión original. Si se acepta el concepto de trayectoria, la restauración sólo es aplicable a muy corto plazo y para perturbaciones locales (Dufour y Piégay, 2009).

Ante estas dificultades se va asistiendo a un cambio progresivo de mentalidad en los defensores de la restauración hacia una posición más posibilista que se ubica cla- 
ramente en el campo de la rehabilitación. En el ámbito fluvial Brierley y Fryirs (2008) llegan a considerar la restauración irreal y utópica, descartando totalmente el término y empleando en exclusiva el de rehabilitación (Fryirs y Brierley, 2009). Al mismo tiempo, se da una importancia fundamental a la naturalidad como clave de gestión ambiental, considerando los caracteres naturales, independientemente de su origen y trayectoria (no confundir lo "natural" con lo actualmente imposible "prístino" o "virgen"), como fuente de beneficios para la sociedad. Se asume además la importancia del papel del hombre en los sistemas naturales y la necesidad de incluir en la recuperación ambiental los valores culturales junto a los naturales. Esta nueva perspectiva es fundamentalmente práctica, en el sentido de que es más fácil que pueda ser ejecutada, más "vendible" y socialmente aceptable. Sin embargo, presenta dos problemas: a) en muchos casos las actuaciones pueden quedarse en un maquillaje muy superficial, recuperándose pocos valores naturales; b) se puede estar favoreciendo que la rehabilitación se convierta ante todo en un buen negocio o bien en un instrumento de promoción política.

\subsection{El ejemplo de la restauración fluvial}

La restauración fluvial se ha convertido en los últimos años en la punta de lanza científica sobre la restauración y rehabilitación ambiental. Se mantiene vivo un intenso y valioso debate sobre los objetivos y la viabilidad de la restauración fluvial con aportaciones como las de Williams (2001), Shields et al. (2003), Rohde (2004), Hughes et al. (2005), Newson y Large (2006), Palmer et al. (2007, 2010), Junker y Bucheker (2008), Fryirs y Brierley (2009) ó Dufour y Piégay (2009).

La auténtica restauración fluvial debería ser fundamentalmente auto-restauración hidro-geomorfológica y requeriría caudales naturales incluyendo crecidas, sedimentos movilizables, espacio (territorio fluvial) para el desarrollo de la dinámica natural, eliminar obstáculos longitudinales y transversales, y tiempo para la auto-recuperación. Sin embargo, esta auténtica restauración es imposible en la práctica, ya que los ríos, torrentes, barrancos y ramblas sufren numerosas presiones en toda su cuenca, que en su mayoría no se pueden eliminar. Si fuera posible eliminar todos esos impactos (presas, defensas, canalizaciones), la restauración sería rápida y efectiva en muchos ríos, ya que unas pocas crecidas serían suficientes para recuperar todo el funcionamiento natural.

La sociedad actual consume mucha agua y también sedimentos (áridos) y está ocupando buena parte de los territorios fluviales, intensos procesos de urbanización y especulación que provocan un continuo incremento en el deterioro fluvial. Además, la correcta restauración está muy alejada de las actuales demandas sociales: seguridad, estabilidad, parques fluviales, domesticación de las riberas, modelos urbanos 
frente a naturalidad (gravas y vegetación espontánea se consideran sucias), moda en la construcción de los escollerados innecesarios, etc.

La restauración fluvial se apoyaría en medidas como la prohibición de dragados y "limpiezas", la apertura o derribo de presas para el paso de los sedimentos, la recuperación de caudales naturales y sobre todo de las crecidas dinamizadoras. Asimismo es necesaria la eliminación de defensas para permitir la erosión de las orillas y la removilización de sedimentos, la devolución al cauce de su espacio de movilidad, permitir que se generen nuevas superficies de sedimentos que la vegetación irá colonizando o no, así como dejar que las riberas se vayan estructurando en complejos mosaicos a partir de la dinámica hidrogeomorfológica, permitiendo que regresen valiosas especies autóctonas, etc. En suma, se trata de liberar al sistema fluvial de presiones para que pueda volver a ejercer sus funciones: que el río vuelva a funcionar como un río.

Frente a todo ello se encuentra el pobre sucedáneo que la mayoría de las veces se ejecuta como marketing de restauración: estabilizaciones de orillas (aunque se haga con técnicas blandas de ingeniería natural), revegetaciones, plantaciones de chopos, ajardinamiento y urbanización de márgenes, maquillaje de dragados o escolleras, ampliaciones de sección de desagüe, etc. También hay actuaciones que, para lograr mayor heterogeneidad y biodiversidad, han creado nuevos cauces complejos y meandriformes sobre ríos que en estado natural eran mucho más simples (Fryirs y Brierley, 2009; Palmer et al., 2010).

\section{4. ¿Exigencia ambiental, reto, mito o negocio?}

Restaurar sistemas naturales es prácticamente imposible y rehabilitarlos es una labor científico-técnica muy difícil. Muchos proyectos fracasan porque no han pasado por la fase de eliminar o reducir significativamente las causas de deterioro. Es imposible restaurar algunos grandes impactos y se puede hacer muy poco en su rehabilitación, de manera que hay sistemas naturales irrecuperables (Jungwirth et al., 2002). Hay también notables dificultades técnicas, por la complejidad de los sistemas naturales, la imposibilidad de comprobar a escala humana algunos resultados o la incertidumbre de que extrapolando a otros enclaves se llegue a los mismos resultados. Muchos proyectos se basan en modelos realizados en sistemas naturales diferentes y regiones diferentes. De hecho, en algunos ámbitos (por ejemplo en España, y en ambientes mediterráneos en general) son todavía muy escasas las auténticas restauraciones antecedentes y los buenos ejemplos de rehabilitación que puedan servir como modelo de actuación. De acuerdo con Comín (2002), la práctica de la restauración va mucho más rápida que la teoría, ya que ésta constituye una línea científica relativamente joven. Además, para la restauración es necesaria la aplicación de numerosos conocimientos procedentes de variadas disciplinas científicas. 
En consecuencia, los proyectos suelen basarse en prácticas simples e insuficientes, como la frecuente revegetación. Son programas que se reducen a una visión simplista de reverdecimiento de un área, sin considerar procesos ni interacciones. Las especies no siempre están bien elegidas y casi siempre se subestima la rehabilitación de la geomorfología y del suelo. Esto ocurre con mucha frecuencia cuando la rehabilitación se ejecuta sólo como exigencia ambiental (a raíz de una evaluación de impacto), limitándose a medidas correctoras convencionales y reproducidas "en serie".

Además, en muchos casos hay que vencer enormes inercias sociales y deficiencias administrativas y jurídicas. Cabe añadir la presión temporal con que se desarrollan muchas actividades de restauración o rehabilitación, delimitadas por el período legislativo.

Ante todas estas dificultades, los programas de restauración ambiental constituyen un auténtico reto. Desde amplios sectores conservacionistas y científicos, la restauración se ha elevado a paradigma por el que hay que luchar, a pesar de su complejidad y de lo utópico que se prevé teniendo en cuenta la situación de partida. Adoptando una posición más práctica, para algunos autores la restauración es un mito (Dufour y Piégay, 2009). También puede considerarse la restauración ambiental un negocio al que la administración dedica crecientes presupuestos con escaso control sobre el destino, ya que se financian con frecuencia, bajo el nombre vendible de restauración, actuaciones contrarias a los fines ambientales. Por ello Comín (2002) señala que la restauración es en ocasiones una estrategia de obtención de beneficios económicos, publicidad e imagen, y de orientación de la opinión de personas.

En ocasiones las actuaciones son desproporcionadas o innecesarias. Hay prácticas de restauración que deterioran más que recuperan (Comín, 2002). Las principales empresas que realizan estas "restauraciones" son las mismas de ingeniería civil que ejecutan obras públicas. El simple hecho de utilizar una técnica, aun siendo "blanda", "ecológica" o "verde" no significa que se esté restaurando ni que al proyecto se le pueda llamar restauración. De hecho, muchas de las técnicas se utilizan en actuaciones de objetivo meramente económico o estabilizador, o bien se repiten en diferentes ámbitos de restauración, lo que evidencia que hay pocos diseños específicos y pocas empresas especializadas. Pero para las administraciones es más fácil y correcto financiar actuaciones técnicas concretas, visibles y presupuestables que simples eliminaciones de impactos acompañadas de un seguimiento científico largo que vaya constatando si el sistema natural se va auto-recuperando.

\subsection{Mirando al futuro}

No hay que renunciar de antemano a la restauración ambiental por sus dificultades. Puede ser perfectamente factible y viable en sistemas naturales sencillos afecta- 
dos sólo por impactos locales. Por tanto, deben seguir diseñándose, incluso aunque se presuman muchas dificultades, propuestas y programas de restauración, que pueden más adelante reconvertirse en procesos de rehabilitación si las circunstancias así lo exigen. Ahora bien, en sistemas muy complejos y afectados por múltiples presiones e impactos, es imprescindible una rehabilitación fundamentada en objetivos concretos y ejecutada con acciones concretas. Lo más inteligente puede ser pensar en restauración y actuar en rehabilitación, desarrollar la teoría y los principios de la restauración y, teniéndolos en cuenta, ejecutar adecuadamente las técnicas de rehabilitación, llegando lo más lejos posible en los ideales de la restauración.

Restauración y rehabilitación deben basarse en objetivos hacia el futuro más que en recuperar situaciones pasadas. Si es necesario encontrar un modelo de referencia éste no ha de buscarse en el pasado, sino en otro sistema natural actual próximo que se encuentre en buen estado. Es esencial definir con claridad y precisión los objetivos, que deben resultar de la combinación de lo que se pretende recuperar en el sistema natural y de lo que funcionalmente es posible de acuerdo con su trayectoria y estado. Deben fundamentarse en valores naturales y en caracteres locales. En algunos casos también se puede tener en cuenta lo que la sociedad necesita y económicamente es factible (Aronson et al., 2006), así como aspectos culturales, que no hay por qué subestimar en un proyecto de mejora ambiental.

Es urgente y necesario desarrollar normativas y establecer algún organismo público de tutela de la restauración y de la rehabilitación. Algunas leyes y directivas europeas no tratan de restauración pero sientan bases de apoyo válidas. Paralelamente pueden plantearse moratorias para que no se sigan realizando ciertas actuaciones impactantes, así como endurecer en muchos casos el procedimiento de evaluación de impacto ambiental. Igualmente hay que trabajar en nuevas figuras de ordenación del territorio y planeamiento urbanístico. En suma, es preciso integrar la restauración en planes de gestión más amplios.

La restauración ambiental no puede hacerse depender de presupuestos económicos que respondan a plazos cortos de tiempo. Se trate de restauración o de rehabilitación, supone un proceso generalmente muy largo que requiere seguimiento científico y cuya conclusión no puede ser prevista ni certificada. No pueden ejecutarse intervenciones por el hecho de que sean rápidamente observables y vendibles, ya que probablemente constituirán un fracaso en cuanto a sus objetivos ambientales. El imprescindible seguimiento de los resultados para comprobar la consecución de los objetivos puede implicar en muchas ocasiones el reajuste de los objetivos y de los medios del proceso de restauración o de rehabilitación, en la línea de la gestión adaptativa.

Es muy necesaria la formación técnica y científica en los diferentes ámbitos de restauración. Ante la fuerte demanda profesional que puede preverse, se hace necesario consolidar currículos formativos en educación superior. 
También es fundamental la educación ambiental de la sociedad sobre el funcionamiento de los sistemas naturales y los beneficios y objetivos de los procesos recuperadores. En este sentido, no puede seguir llamándose restauración a lo que no lo es. No hay que engañar a la sociedad tratando de vender ciertas actuaciones con un "envoltorio verde".

\section{Conclusiones}

La interacción del hombre con los sistemas naturales está tan extendida y es tan antigua y compleja que resulta imposible en la práctica devolver a los ecosistemas degradados a una situación previa a las perturbaciones. En la actual sociedad de consumo ejecutar programas de auténtica restauración ambiental implicaría una auténtica revolución en la que habría que modificar hábitos y actividades y eliminar estructuras impactantes. Ante la dificultad de lograr auténticas restauraciones ambientales, es imprescindible reflexionar sobre los conceptos, objetivos y posibilidades de esta potente línea de trabajo, auténtico reto del siglo XXI. Así, parece inevitable renunciar a la restauración y trabajar en la rehabilitación, pero esta renuncia no puede afectar a la teoría, a la ciencia ni a la educación, que deben seguir valorando la auténtica restauración como paradigma y meta final, aunque sea una meta utópica. Solo desde la afirmación de estos valores naturales se puede confiar en que la rehabilitación realista, factible y viable, logre mejoras en los sistemas naturales y no caiga en el puro maquillaje o en el engaño derivado del negocio y la rentabilidad política. El posibilismo de la rehabilitación debe servir también a ese deber final de conservar y mejorar el medio ambiente para traspasarlo a las generaciones futuras en buen estado.

Desde la ciencia hay que seguir creyendo en el paradigma de la restauración ambiental, desarrollando sus bases teóricas e investigando sobre el funcionamiento de los sistemas naturales, y también hay que colaborar en los trabajos de rehabilitación, tratando de lograr objetivos ambientales adecuados y lo más ambiciosos que sea posible, desde el seguimiento y la gestión adaptativa en cada proyecto. También es urgente y necesario trabajar en la formación de los futuros gestores, técnicos y profesionales, así como en la educación de la sociedad y en la denuncia de las falsas restauraciones. A la administración hay que exigir responsabilidad en estos campos, que desarrolle normativas y que establezca algún sistema de tutela y evaluación de proyectos. 


\section{Referencias}

Aronson, J.; Clewell, A.F.; Blignaut, J.N. y Milton, S.J. (2006). Ecological restoration: a new frontier for nature conservation and economics. Journal for Nature Conservation, 14, 135-139.

Brierley, G.J. y Fryirs, K.A. (2008). Moves toward an era of river repair. In Brierley, G.J. y Fryirs, K.A. (Eds.) River futures. An integrative scientific approach to river repair, 3-15, Washington, Island Press.

Comín, F.A. (2002). Restauración ecológica: teoría versus práctica. Ecosistemas, XI(1). 11-13.

Dufour, S. y Piégay, H. (2009). From the myth of a lost paradise to targeted river restoration: forget natural references and focus on human benefits. River Research and Applications, 25, 568-581.

Elliot, R. (1982). Faking Nature. Inquiry, 25, 81-93.

Fryirs, K. y Brierley, G.J. (2009). Naturalness and place in river rehabilitation. Ecology and Society, 14(1), 20. (online)

Gómez Orea, D. (Dir., 2004). Recuperación de espacios degradados. Madrid, Mundiprensa.

González del Tánago, M. y García de Jalón, D. (2007). Restauración de ríos. Guía metodológica para la elaboración de proyectos. Madrid, Ministerio de Medio Ambiente.

Herrera, A. (2008). Disertación crítica sobre la evaluación del estado ecológico de las masas de agua y los proyectos de restauración, recuperación o mejora ambiental de cauces: del mito a la realidad. $V I$ Congreso Ibérico sobre Gestión y Planificación del Agua. Vitoria-Gasteiz, Fundación Nueva Cultura del Agua.
Hughes, F.M.R.; Colston, A. y Mountford, J.O. (2005). Restoring riparian ecosystems: the challenge of accommodating variability and designing restoration trajectories. Ecology and Society, 10(1), 12 (online)

Jungwirth, M.; Muhar, S. y Schmutz, S. (2002). Re-establishing and assessing ecological integrity in riverine landscapes. Freshwater Biology, 47, 867-887.

Junker, B. y Buchecker, M. (2008). Aesthetic preferences versus ecological objectives in river restorations. Landscape and Urban Planning, 85(3-4), 141-154.

Katz, E. (2003). La gran mentira: la restauración humana de la naturaleza. In Kwiatkowska, T. e Issa, J. (Coords.) Los caminos de la ética ambiental, II, 233-244, México, Plaza y Valdés.

Muhar, S., Schmutz, S. y Jungwirth, M., (1995). River restoration concepts, goals and perspectives. Hydrobiologia, 303, 183-194

Newson, M.D. y Large, A.R.G. (2006). 'Natural' rivers, 'hydromorphological quality' and river restoration: a challenging new agenda for applied fluvial geomorphology. Earth Surface Processes and Landforms, 31, 1606-1624.

Palmer, M.A.; Allan, J.D.; Meyer, J. y Bernhardt, E.S. (2007). River restoration in the twenty-first century: data and experiential knowledge to inform future efforts. Restoration Ecology, 15(3), 472-481.

Palmer, M.; Menninger, H.L. y Bernhardt, E.S (2010). River restoration, habitat heterogeneity and biodiversity: a failure of theory or practice? Freshwater Biology, 55(1), 205-222.

Rohde, S. (2004). River restoration: potential and limitations to re-establish riparian 
landscapes. Assessment and planning. PhD dissertation, Zürich, Swiss Federal Institut of Technology.

Sánchez, O. (2005). Restauración ecológica: algunos conceptos, postulados y debates al inicio del siglo XXI. In: Sánchez, O.; Peters, E.; Márquez-Huitzil, R.; Vega, E.; Portales, G.; Valdez, M. y Azuara, D. (Eds.). Temas sobre restauración ecológica, 15-30. México, Inst. Nacional de Ecología.

Shields, F.D.Jr.; Cooper, C.M.; Knight, S.S. y Moore, M.T. (2003). Stream corridor restoration research: a long and winding road. Ecological Engineering, 20, 441-454.
Soar, P.J. y Thorne, C.R. (2001). Channel restoration design for meandering rivers. Washington, Engineer Research and Development Center. U.S.Army Corps of Engineers

Turner, M.G.; Romme, W.H.; Gardner, R.H. O’Neill, R.V. y Kratz, T.K. (1993). A revised concept of landscape equilibrium: disturbance and stability on scaled landscapes. Landscape Ecology, 8(3), 213-227.

Williams, P.B. (2001). River engineering versus river restoration. In Hayes, D.F. (Ed.) $A S C E$ Wetlands Engineering \& River Restoration Conference, Reno, American Society of Civil Engineers. 